\title{
ON QUASI-REPRESENTING GRAPHS FOR A CLASS OF $\mathcal{B}^{(1)}{ }_{\text {-GROUPS }}$
}

\author{
Peter Dongunn Yom
}

\begin{abstract}
In this article, we give a characterization theorem for a class of corank-1 Butler groups of the form $\mathcal{G}\left(A_{1}, \ldots, A_{n}\right)$. In particular, two groups $G$ and $H$ are quasi-isomorphic if and only if there is a labelpreserving bijection $\phi$ from the edges of $T$ to the edges of $U$ such that $S$ is a circuit in $T$ if and only if $\phi(S)$ is a circuit in $U$, where $T, U$ are quasi-representing graphs for $G, H$ respectively.
\end{abstract}

The terminology group in this article means a torsion-free abelian group. A Butler group is a pure subgroup of a finite rank completely decomposable group and a $\mathcal{B}^{(1)}$-group is a Butler group of the form $C / X$ where $C$ is a finite rank completely decomposable group and $X$ a rank one pure subgroup of $C$. Let $A_{1}, \ldots, A_{n}(n \geq 2)$ be nonzero subgroups of $\mathbb{Q}$ and we will consider $\mathcal{B}^{(1)}$-groups of the form $\mathcal{G}\left(A_{1}, \ldots, A_{n}\right)=$ the kernel of the codiagonal map $\bigoplus_{i=1}^{n} A_{i} \rightarrow \mathbb{Q}$ given by $\left(a_{1}, \ldots, a_{n}\right) \mapsto \sum a_{i}$. The groups $\mathcal{G}\left(A_{1}, \ldots, A_{n}\right)$ and their dual groups $\mathcal{G}\left[A_{1}, \ldots, A_{n}\right]$ have been studied extensively and classified up to numerical quasi-isomorphism and isomorphism invariants by various authors since F. Richman classified a relatively small family of Butler groups that he called 'doubly incomparable' in [5]. A representing graph for $\mathcal{G}\left(A_{1}, \ldots, A_{n}\right)$ was first introduced by D. Arnold and C. Vinsonhaler in [1] and it was used to obtain numerical quasi-isomorphism invariants for classes of CT-groups [2] and strongly indecomposable groups [3]. In this article, we give a characterization theorem for a class of groups $\mathcal{G}\left(A_{1}, \ldots, A_{n}\right)$ in terms of quasi-representing graphs.

\section{Quasi-representing graphs}

We define type as an isomorphism class of subgroups of the additive group of rationals $\mathbb{Q}$. Let $\tau_{i}=$ type $A_{i}$ for each $1 \leq i \leq n$ and we shall refer to the group $G=\mathcal{G}\left(A_{1}, \ldots, A_{n}\right)$ as $G=\mathcal{G}\left(\tau_{1}, \ldots, \tau_{n}\right)$, keeping in mind that $G$ is defined up to quasi-isomorphism. Let $G=\mathcal{G}\left(\tau_{1}, \ldots, \tau_{n}\right)$ and define $\mathcal{C}_{G}$ to

Received May 30, 2010.

2010 Mathematics Subject Classification. Primary 20K15.

Key words and phrases. Butler groups, $\mathcal{B}^{(1)}$-groups, quasi-representing graphs, quasiisomorphisms. 
be the complete graph with vertices $\tau_{1}, \ldots, \tau_{n}$ and edges $\tau_{i} \tau_{j}$ labelled by types $\tau_{i} \wedge \tau_{j}$ for $1 \leq i \neq j \leq n$. A representing graph for $G$ is any subgraph of $\mathcal{C}_{G}$ that is obtained by iteration of the algorithm: if a graph contains a circuit $S$ with all the edges labelled with types $\geq \tau$ and at least one edge labelled with $\tau$, then remove an edge of $S$ labelled by $\tau$. A labelled graph is a quasirepresenting graph for a Butler groups $H$ if it is a representing graph for some group $\mathcal{G}\left(\sigma_{1}, \ldots, \sigma_{n}\right)$ which is quasi-isomorphic to $H$. Suppose $T$ is a quasirepresenting graph for a Butler group $H$, with $n$ vertices and with edges $\tau_{i} \tau_{j}$ labelled with types $\tau_{i} \wedge \tau_{j}$. If we let $\sigma_{i}=\bigvee\left\{\tau_{i} \wedge \tau_{j}: \tau_{i} \tau_{j}\right.$ is an edge in $T$ and $j \neq i\}$ for each $1 \leq i \leq n$, then $T$ is a representing graph for $\mathcal{G}\left(\sigma_{1}, \ldots, \sigma_{n}\right)$ and $\mathcal{G}\left(\sigma_{1}, \ldots, \sigma_{n}\right)$ is quasi-isomorphic to $H$. The detail of representing graphs can be found in [1].

Notation. Two groups $G$ and $H$ are quasi-isomorphic, $G \dot{\sim} H$, if $G$ is isomorphic to a subgroup of finite index in $H$. For any nonempty subset $I$ of $\left\{\tau_{1}, \ldots, \tau_{n}\right\}$, we denote $\tau^{I}=\bigvee_{\tau_{i} \in I} \tau_{i}$.

Definition 1.1. We say that subsets $X, Y$ of $\left\{\tau_{1}, \ldots, \tau_{n}\right\}$ is a TVE-partition (two-vertex exchange partition) for $\tau_{i}, \tau_{j}$ if $X \cap Y=\emptyset, X \cup Y=\left\{\tau_{1}, \ldots, \tau_{n}\right\} \backslash$ $\left\{\tau_{i}, \tau_{j}\right\}$ and $\tau^{X} \wedge \tau^{Y} \leq \tau_{i} \wedge \tau_{j}$.

A collection of types $\tau_{1}, \ldots, \tau_{n}$ is called trimmed if $\tau_{i} \leq \bigvee_{j \neq i} \tau_{j}$ for each $1 \leq i \leq n$. Throughout the article we assume, unless otherwise stated, all collections of types $\tau_{1}, \ldots, \tau_{n}$ are trimmed. The following lemma is Theorem 4 in [7].

Lemma 1.1 ('two-vertex exchange'). Let $\tau_{1}, \ldots, \tau_{n}$ be trimmed and $\tau_{i} \neq \sigma_{j}$ for $i, j \in\{1,2\}$. Then the following statements are equivalent:

(a) $\mathcal{G}\left(\tau_{1}, \tau_{2}, \tau_{3}, \ldots, \tau_{n}\right) \dot{\sim} \mathcal{G}\left(\sigma_{1}, \sigma_{2}, \tau_{3}, \ldots, \tau_{n}\right)$;

(b) There is a TVE-partition $X, Y$ for $\tau_{1}, \tau_{2}$ and $\sigma_{1}=\left(\tau_{1} \vee \tau^{Y}\right) \wedge\left(\tau_{2} \vee \tau^{X}\right)$ and $\sigma_{2}=\left(\tau_{1} \vee \tau^{X}\right) \wedge\left(\tau_{2} \vee \tau^{Y}\right)$.

Suppose $X, Y$ is a TVE-partition for $\tau_{1}, \tau_{2}$ in $G=\mathcal{G}\left(\tau_{1}, \ldots, \tau_{n}\right)$ and $\sigma_{1}, \sigma_{2}$ defined as in Lemma 1.1 then we say $H=\mathcal{G}\left(\sigma_{1}, \sigma_{2}, \tau_{3}, \ldots, \tau_{n}\right)$ is obtained from $G$ by a two-vertex exchange and we will write $H=\left(G ; X, Y ; \tau_{1}, \tau_{2}\right)$.

Notation. If $X, Y$ is a TVE-partition for $\tau_{1}, \tau_{2}$ in $G=\mathcal{G}\left(\tau_{1}, \ldots, \tau_{n}\right)$ and $H=\left(G ; X, Y ; \tau_{1}, \tau_{2}\right)$, then, for notational convenience, we let $\hat{\tau}_{1}=\left(\tau_{1} \vee \tau^{Y}\right) \wedge$ $\left(\tau_{2} \vee \tau^{X}\right)$ and $\hat{\tau}_{2}=\left(\tau_{1} \vee \tau^{X}\right) \wedge\left(\tau_{2} \vee \tau^{Y}\right)$ and $H=\mathcal{G}\left(\hat{\tau}_{1}, \hat{\tau}_{2}, \tau_{3}, \ldots, \tau_{n}\right)$.

Since the lattice of types is distributive, we can show that

$$
\tau_{1} \wedge \tau_{2}=\hat{\tau}_{1} \wedge \hat{\tau}_{2} ; \tau_{1} \wedge \tau_{k}=\hat{\tau}_{1} \wedge \tau_{k} \text { and } \tau_{2} \wedge \tau_{k}=\hat{\tau}_{2} \wedge \tau_{k} \text { if } \tau_{k} \in X
$$

Let $\mathcal{C}_{G}$ be the complete graph with vertices $\tau_{1}, \ldots, \tau_{n}$ and each edge $\tau_{i} \tau_{j}$ labelled with type $\tau_{i} \wedge \tau_{j}$ for $1 \leq i \neq j \leq n$. Using (1) we define a label-preserving bijection $\phi$ from the edges of $\mathcal{C}_{G}$ to the edges of $\mathcal{C}_{H}$ as follows:

$$
\phi\left(\tau_{i} \tau_{j}\right)=\tau_{i} \tau_{j} \text { for } 3 \leq i \neq j \leq n \text { and } \phi\left(\tau_{1} \tau_{2}\right)=\hat{\tau}_{1} \hat{\tau}_{2} ;
$$




$$
\begin{array}{r}
\phi\left(\tau_{1} \tau_{k}\right)=\hat{\tau}_{1} \tau_{k} \text { and } \phi\left(\tau_{2} \tau_{k}\right)=\hat{\tau}_{2} \tau_{k} \text { if } \tau_{k} \in X ; \\
\phi\left(\tau_{2} \tau_{m}\right)=\hat{\tau}_{1} \tau_{m} \text { and } \phi\left(\tau_{1} \tau_{m}\right)=\hat{\tau}_{2} \tau_{m} \text { if } \tau_{m} \in Y .
\end{array}
$$

Definition 1.2. Let $G=\mathcal{G}\left(\tau_{1}, \ldots, \tau_{n}\right)$.

(a) $\phi: \mathcal{C}_{G} \rightarrow \mathcal{C}_{H}$ is called a TVE-map if $H=\mathcal{G}\left(\hat{\tau}_{1}, \hat{\tau}_{2}, \tau_{3}, \ldots, \tau_{n}\right)=$ $\left(G ; X, Y ; \tau_{1}, \tau_{2}\right)$ and $\phi$ is a label-preserving bijection map from the edges of $\mathcal{C}_{G}$ to the edges of $\mathcal{C}_{H}$ defined as in (2) above.

(b) Let $A$ and $B$ be labelled graphs then we say $\psi: A \rightarrow B$ is a $C L P$-map if $\psi$ is a label-preserving bijection from the edges of $A$ to the edges of $B$ such that $S$ is a circuit in $A$ if and only if $\psi(S)$ is a circuit in $B$.

Let $\mathcal{Q}(G)$ be the set of all quasi-representing graphs for $G$.

Lemma 1.2. Let $G=\mathcal{G}\left(\tau_{1}, \ldots, \tau_{n}\right)$ and $H=\mathcal{G}\left(\sigma_{1}, \ldots, \sigma_{n}\right)$. If $\psi: T \rightarrow U$ is a CLP-map for some $T \in \mathcal{Q}(G)$ and $U \in \mathcal{Q}(H)$, then $G \dot{\sim} H$.

Proof. Let $T \in \mathcal{Q}(G)$ and $U \in \mathcal{Q}(H)$ and $\psi: T \rightarrow U$ be a CLP-map. Then there is a group isomorphism from $D_{T}$ to $D_{U}$ sending $K_{T}$ to $K_{U}$, where $D_{T}=$ $\bigoplus\left\{\tau_{i} \wedge \tau_{j}: \tau_{i} \tau_{j}\right.$ is an edge in $\left.T\right\}$ and $K_{T}$ is the pure subgroup of $D_{T}$ generated by the circuits of $T$ by Proposition 2.1 in [2]. Since $G \dot{\simeq} D_{T} / K_{T}$ and $H \dot{\sim} D_{U} / K_{U}$ by Corollary 1.7 in [1], it follows that $G \dot{\sim} H$.

Let $T \in \mathcal{Q}(G)$. For a type $\tau$, define $T(\tau)$ be the subgraph of $T$ whose edges are labelled with types $\geq \tau$.

Lemma 1.3 (Lemma 1 in [3]). Let $G=\mathcal{G}\left(\tau_{1}, \ldots, \tau_{n}\right)$ and $T \in \mathcal{Q}(G)$ and suppose $\tau_{i}, \tau_{j}, \tau_{k}$ are distinct vertices of $T$ with $\tau_{i} \wedge \tau_{j} \leq \tau_{k}$. If edge $\tau_{i} \tau_{j} \in T$ with type $\tau=\tau_{i} \wedge \tau_{j}$, then there is a path $P$ in $T(\tau) \backslash\left\{\tau_{i} \tau_{j}\right\}$ connecting either $\tau_{i}$ or $\tau_{j}$ to $\tau_{k}$. If $P$ connects $\tau_{i}$ (respectively, $\tau_{j}$ ) to $\tau_{k}$, then $\tau_{i} \tau_{j}$ may be replaced by $\tau_{k} \tau_{j}$ (respectively, $\left.\tau_{k} \tau_{i}\right)$ to obtain a new quasi-representing graph for $G$ and $\tau_{i} \wedge \tau_{j}=\tau_{k} \wedge \tau_{j}\left(\right.$ respectively, $\left.\tau_{k} \wedge \tau_{i}\right)$.

Remark 1.1. Observe that in Lemma 1.3 there are no two paths $P$ and $P^{\prime}$ in $T(\tau) \backslash\left\{\tau_{i} \tau_{j}\right\}$ such that $P$ connects $\tau_{i}$ to $\tau_{k}$ and $P^{\prime}$ connects $\tau_{j}$ to $\tau_{k}$. If both $P$ and $P^{\prime}$ exist, then $P \cup P^{\prime} \cup\left\{\tau_{i} \tau_{j}\right\}$ contains a circuit $S$ such that $\tau_{i} \tau_{j}$ is an edge in $S$ and $S \subseteq T(\tau)$ where $\tau=\tau_{i} \wedge \tau_{j}$, a contradiction to the fact that $T$ is a quasi-representing graph.

Notation. Let $G=\mathcal{G}\left(\tau_{1}, \ldots, \tau_{n}\right)$ and $T \in \mathcal{Q}(G)$. We write $\tau_{i} \tau_{j} \rightarrow \tau_{k} \tau_{m}$ if $\tau_{i} \wedge$ $\tau_{j}=\tau_{k} \wedge \tau_{m}$ and the edge $\tau_{i} \tau_{j} \in T$ is replaced by the edge $\tau_{k} \tau_{m}$ to obtain a new quasi-representing graph $T^{\prime}$ for $G$, that is, $T^{\prime}=\left(T \backslash\left\{\tau_{i} \tau_{j}\right\}\right) \cup\left\{\tau_{k} \tau_{m}\right\} \in \mathcal{Q}(G)$. We also write $\tau_{k} \tau_{m} \rightarrow \tau_{k} \tau_{r}$ or $\tau_{m} \tau_{r}$ if either $\tau_{k} \tau_{m} \rightarrow \tau_{k} \tau_{r}$ or $\tau_{k} \tau_{m} \rightarrow \tau_{m} \tau_{r}$.

Lemma 1.4. Suppose $X, Y$ is a TVE-partition for $\tau_{1}, \tau_{2}$ in $G=\mathcal{G}\left(\tau_{1}, \ldots, \tau_{n}\right)$ and let $E=\left\{\tau_{k} \tau_{m}: \tau_{k} \tau_{m}\right.$ is an edge and $\tau_{k} \wedge \tau_{m} \leq \tau_{1} \wedge \tau_{2}$ for $\left.3 \leq k \neq m \leq n\right\}$ and $F=\left\{\tau_{k} \tau_{m}: \tau_{k} \tau_{m}\right.$ is an edge and $\tau_{k} \wedge \tau_{m} \leq \tau_{t}$ where $\tau_{k}, \tau_{m} \in X$ and $\left.\tau_{t} \in Y\right\}$, then there exists $T \in \mathcal{Q}(G)$ such that $T \subseteq \mathcal{C}_{G} \backslash(E \cup F)$. 
Proof. Let $X, Y$ be a TVE-partition for $\tau_{1}, \tau_{2}$ in $G=\mathcal{G}\left(\tau_{1}, \ldots, \tau_{n}\right)$ and $V \in$ $\mathcal{Q}(G)$. If edge $\tau_{k} \tau_{m} \in V \cap E$, then $\tau_{k} \tau_{m} \rightarrow \tau_{k} \tau_{r}$ or $\tau_{m} \tau_{r}$ for $r \in\{1,2\}$ by Lemma 1.3 and similarly, we can replace all other edges in $V \cap E$ to obtain $V^{\prime} \in \mathcal{Q}(G)$ such that $V^{\prime} \subseteq \mathcal{C}_{G} \backslash E$. If edge $\tau_{k} \tau_{m} \in V^{\prime} \cap F$, then $\tau_{k} \tau_{m} \rightarrow \tau_{k} \tau_{t}$ or $\tau_{m} \tau_{t}$ by Lemma 1.3. Without loss of generality, assume $\tau_{k} \tau_{m} \rightarrow \tau_{k} \tau_{t}$. Since $X, Y$ is the TVE-partition for $\tau_{1}, \tau_{2}$ in $G$, it follows that $\tau^{X} \wedge \tau^{Y} \leq \tau_{1} \wedge \tau_{2}$ and consequently, $\tau_{k} \wedge \tau_{t} \leq \tau_{r}$ and $\tau_{k} \tau_{t} \rightarrow \tau_{k} \tau_{r}$ or $\tau_{r} \tau_{t}$ for $r \in\{1,2\}$. With a similar argument, we can remove all edges in $V^{\prime} \cap F$ to obtain $T \in \mathcal{Q}(G)$ such that $T \subseteq \mathcal{C}_{G} \backslash(E \cup F)$.

Notation. Let $S$ be a subgraph of $T$ and we denote $S_{V}=$ the set of all vertices in $S$ and $e(A, B)_{T}=\left\{\tau_{i} \tau_{j}: \tau_{i} \tau_{j}\right.$ is an edge in $T$ and $\left.\tau_{i} \in A, \tau_{j} \in B\right\}$ for $A, B \subseteq T_{V}$.

In the proposition below, we list some results from [7].

Proposition 1.5. Let $G=\mathcal{G}\left(\tau_{1}, \ldots, \tau_{n}\right)$ and suppose $X, Y$ is a TVE-partition for $\tau_{1}, \tau_{2}$ in $G$. Then

(a) Let $T \in \mathcal{Q}(G)$ such that $e(X, Y)_{T}=\emptyset$ and if $H=\left(G ; X, Y ; \tau_{1}, \tau_{2}\right)$ and $\phi: \mathcal{C}_{G} \rightarrow \mathcal{C}_{H}$ is a TVE-map, then $U=\phi(T) \in \mathcal{Q}(H)$ and $\left.\phi\right|_{T}: T \rightarrow U$ is a CLP-map.

(b) If $\tau_{1}, \tau_{2}, \tau_{3}, \ldots, \tau_{n}$ is trimmed, then

(i) $\hat{\tau}_{1}, \hat{\tau}_{2}, \tau_{3}, \ldots, \tau_{n}$ is trimmed and $\tau_{1} \wedge \tau_{2}=\hat{\tau}_{1} \wedge \hat{\tau}_{2}, \tau_{1}=\left(\hat{\tau}_{1} \vee \tau^{Y}\right) \wedge$ $\left(\hat{\tau}_{2} \vee \tau^{X}\right)$ and $\tau_{2}=\left(\hat{\tau}_{1} \vee \tau^{X}\right) \wedge\left(\hat{\tau}_{2} \vee \tau^{Y}\right)$

(ii) $X, Y$ is also a TVE-partition for $\hat{\tau}_{1}, \hat{\tau}_{2}$ in $H$ and $G=(H ; X, Y$; $\left.\hat{\tau}_{1}, \hat{\tau}_{2}\right)$.

\section{CLP-maps}

We say $T \in \mathcal{Q}(G)$ is reduced if (i) for any two edges $e, f$ in $T$ there is a circuit containing both edges $e, f$ and (ii) if label $e \leq$ label $f$, then there is a circuit containing $e$ but not $f$. It is shown that $G$ is strongly indecomposable if and only if each $T \in \mathcal{Q}(G)$ is reduced (Theorem 3 in [3]).

Lemma 2.1. Let $G$ be strongly indecomposable and $T \in \mathcal{Q}(G)$. Then each vertex in $T$ has degree at least two and $\left|e\left(S_{V},(T \backslash S)_{V}\right)_{T}\right| \geq 2$ for any nonempty subgraph $S \subset T$.

Proof. Suppose $G$ is strongly indecomposable and $T \in \mathcal{Q}(G)$ then $T$ is reduced. Since there is a circuit containing any two edges in $T$, each vertex in $T$ has degree at least two. That is, there are at least two edges incident to each vertex in $T$. Thus, we have $\left|e\left(S_{V},(T \backslash S)_{V}\right)_{T}\right| \geq 2$ for any non-empty subgraph $S \subset T$.

We next investigate a relationship between two TVE-partitions.

Lemma 2.2. Let $H=\mathcal{G}\left(\sigma_{1}, \ldots, \sigma_{n}\right)=\mathcal{G}\left(\hat{\tau}_{1}, \hat{\tau}_{2}, \tau_{3}, \ldots, \tau_{n}\right)=\left(G ; X, Y ; \tau_{1}, \tau_{2}\right)$ be strongly indecomposable and let $X^{\prime}, Y^{\prime}$ be a TVE-partition for $\sigma_{i}, \sigma_{j}$ in $H$. Then 
(a) If $\sigma_{t} \neq \hat{\tau}_{t^{\prime}}$ for $t \in\{i, j\}, t^{\prime} \in\{1,2\}$ and $\left\{\sigma_{i}, \sigma_{j}\right\} \subseteq Y$ (respectively, $X$ ), then $\left\{\hat{\tau}_{1}, \hat{\tau_{2}}\right\} \cup X \subseteq X^{\prime}$ or $Y^{\prime}$ (respectively, $\left\{\hat{\tau}_{1}, \hat{\tau_{2}}\right\} \cup Y \subseteq Y^{\prime}$ or $X^{\prime}$ ).

(b) If $\sigma_{i}=\hat{\tau}_{1}, \sigma_{j} \neq \hat{\tau}_{2}$ and $\sigma_{j} \in Y$ (respectively, $X$ ), then $\left\{\hat{\tau}_{2}\right\} \cup X \subseteq X^{\prime}$ or $Y^{\prime}$ (respectively, $\left\{\hat{\tau}_{2}\right\} \cup Y \subseteq Y^{\prime}$ or $\left.X^{\prime}\right)$.

Proof. By Lemma 1.4, there exists $T \in \mathcal{Q}(G)$ such that $T \subseteq \mathcal{C}_{G} \backslash(E \cup F)$ where $E=\left\{\tau_{k} \tau_{m}: \tau_{k} \tau_{m}\right.$ is an edge and $\tau_{k} \wedge \tau_{m} \leq \tau_{1} \wedge \tau_{2}$ for $\left.3 \leq k \neq m \leq n\right\}$ and $F=\left\{\tau_{k} \tau_{m}: \tau_{k} \tau_{m}\right.$ is an edge and $\tau_{k} \wedge \tau_{m} \leq \tau_{t}$ where $\tau_{k}, \tau_{m} \in X$ and $\left.\tau_{t} \in Y\right\}$. If $U=\phi(T) \in \mathcal{Q}(H)$ as in Proposition 1.5(a), then $U \subseteq \mathcal{C}_{H} \backslash(E \cup F)$ because $\sigma_{i}=\tau_{i}$ if $\sigma_{i} \neq \hat{\tau}_{1}$ or $\hat{\tau}_{2}$. For notational convenience, after rearranging the indices of $H$, we let $\sigma_{1}=\sigma_{i}$ and $\sigma_{2}=\sigma_{j}$ and $X^{\prime}, Y^{\prime}$ be the TVE-partition for $\sigma_{1}, \sigma_{2}$ throughout the proof.

(a) Suppose $\left\{\sigma_{1}, \sigma_{2}\right\} \subseteq Y$ and $\sigma_{t} \neq \hat{\tau}_{t^{\prime}}$ for $t, t^{\prime} \in\{1,2\}$. We first show $X \subset X^{\prime}$. Let $A_{1}=X \cap X^{\prime}$ and $A_{2}=X \cap Y^{\prime}$ and suppose $\sigma_{k} \in A_{1}$ and $\sigma_{m} \in A_{2}$. First note that $e(X, Y)_{U}=\emptyset$ and $e\left(A_{1}, A_{2}\right)_{U}=\emptyset$ because $U \subseteq \mathcal{C}_{H} \backslash(E \cup F)$. Let $r \in\{1,2\}$. If $\hat{\tau}_{r} \in X^{\prime}$, then $\hat{\tau}_{r} \sigma_{m} \rightarrow \sigma_{1} \hat{\tau}_{r}$ or $\sigma_{1} \sigma_{m}$ for all $\sigma_{m} \in A_{2}$ and if $\hat{\tau}_{r} \in Y^{\prime}$, then $\hat{\tau}_{r} \sigma_{k} \rightarrow \sigma_{1} \hat{\tau}_{r}$ or $\sigma_{1} \sigma_{k}$ for all $\sigma_{k} \in A_{1}$. Hence, there exists $U^{\prime} \in \mathcal{Q}(H)$ such that (i) $e\left(\hat{\tau}_{r}, A_{2}\right)_{U^{\prime}}=\emptyset$ if $\hat{\tau}_{r} \in X^{\prime}$ (ii) $e\left(\hat{\tau}_{r}, A_{1}\right)_{U^{\prime}}=\emptyset$ if $\hat{\tau}_{r} \in Y^{\prime}$ (iii) $e\left(A_{1}, A_{2}\right)_{U^{\prime}}=\emptyset$ and $e(X, Y)_{U^{\prime}}=\emptyset$. We will show that $U^{\prime}$ does not exist and we conclude that either $A_{1}=\emptyset$ or $A_{2}=\emptyset$.

Suppose $U^{\prime}$ exists and $\left\{\hat{\tau}_{1}, \hat{\tau}_{2}\right\} \subseteq X^{\prime}$ then $e\left(\left\{\hat{\tau}_{1}, \hat{\tau}_{2}\right\}, A_{2}\right)_{U^{\prime}}=\emptyset$. Let $\sigma_{m} \in$ $A_{2}$. If edge $\sigma_{1} \sigma_{m}$ is in $U^{\prime}$, then there is no circuit in $U^{\prime}$ containing $\sigma_{1} \sigma_{m}$ and any edge in $U_{V}^{\prime} \backslash A_{2}$ because $e\left(A_{2}, U_{V}^{\prime} \backslash A_{2}\right)_{U^{\prime}}=e\left(\sigma_{1}, A_{2}\right)_{U^{\prime}}$, a contradiction to the fact $U^{\prime}$ is reduced. If $\sigma_{1} \sigma_{m} \notin U^{\prime}$, then $e\left(A_{2}, U_{V}^{\prime} \backslash A_{2}\right)_{U^{\prime}}=\emptyset$, a contradiction by Lemma 2.1. Hence we must have $\left\{\hat{\tau}_{1}, \hat{\tau}_{2}\right\} \nsubseteq X^{\prime}$ and similarly we can show that $\left\{\hat{\tau}_{1}, \hat{\tau}_{2}\right\} \nsubseteq Y^{\prime}$. Therefore, we assume $\hat{\tau}_{1} \in X^{\prime}$ and $\hat{\tau}_{2} \in Y^{\prime}$ in $U$.

If $\hat{\tau}_{1} \in X^{\prime}$ and $\hat{\tau}_{2} \in Y^{\prime}$ in $U$, then $e\left(\hat{\tau}_{1}, A_{2}\right)_{U^{\prime}}=\emptyset$ and $e\left(\hat{\tau}_{2}, A_{1}\right)_{U^{\prime}}=\emptyset$. Let $\sigma_{k} \in A_{1}$ and $\sigma_{m} \in A_{2}$. If $\sigma_{1} \sigma_{m} \in U^{\prime}$, then $\sigma_{1} \sigma_{m} \rightarrow \hat{\tau}_{2} \sigma_{m}$ or $\sigma_{1} \hat{\tau}_{2}$ because $\sigma_{1} \wedge \sigma_{m} \leq \hat{\tau}_{1} \wedge \hat{\tau_{2}}$ and similarly if $\sigma_{1} \sigma_{k} \in U^{\prime}$, then $\sigma_{1} \sigma_{k} \rightarrow \hat{\tau}_{1} \sigma_{k}$ or $\sigma_{1} \hat{\tau}_{1}$. So, there exists $W \in \mathcal{Q}(H)$ such that $e\left(\sigma_{1}, A_{1} \cup A_{2}\right)_{W}=\emptyset$, but there is no circuit containing edges $\hat{\tau}_{1} \sigma_{k}$ and $\hat{\tau}_{2} \sigma_{m}$ in $W$ because $e\left(A_{1}, A_{2}\right)_{W}=\emptyset$ and $e(X, Y)_{W}=\emptyset$, a contradiction. Since $\hat{\tau}_{t} \neq \sigma_{t^{\prime}}$ for $t, t^{\prime} \in\{1,2\}$, we must conclude that $U^{\prime}$ does not exist and either $A_{1}=\emptyset$ or $A_{2}=\emptyset$. Without loss of generality, we assume $A_{2}=\emptyset$ and $X \subseteq X^{\prime}$ in $U$ and we next show that $\left\{\hat{\tau}_{1}, \hat{\tau}_{2}\right\} \subseteq X^{\prime}$ in $U$.

Suppose $X \subseteq X^{\prime}$ and $\left\{\hat{\tau}_{1}, \hat{\tau}_{2}\right\} \subseteq Y^{\prime}$ in $U$ and if $e\left(\left\{\hat{\tau}_{1}, \hat{\tau}_{2}\right\}, X\right)_{U} \neq \emptyset$, then $\hat{\tau_{r}} \sigma_{k} \rightarrow \sigma_{1} \sigma_{k}$ or $\hat{\tau_{r}} \sigma_{1}$ for some $\sigma_{k} \in X$ and $r \in\{1,2\}$ to obtain $W \in \mathcal{Q}(H)$ such that $e\left(\left\{\hat{\tau}_{1}, \hat{\tau}_{2}\right\}, X\right)_{W}=\emptyset$ and $e(X, Y)_{W}=e\left(\sigma_{1}, X\right)_{W}$ or $e(X, Y)_{W}=\emptyset$. If $\sigma_{1} \sigma_{k} \in W$ for some $\sigma_{k} \in X$, then there is no circuit containing edges $\sigma_{1} \sigma_{k}$ and $\hat{\tau_{1}} \sigma_{t}$ for some $\sigma_{t} \in Y$, a contradiction. If $\sigma_{1} \sigma_{k} \notin W$, then $e\left(X, W_{V} \backslash X\right)_{W}=\emptyset$, a contradiction by Lemma 2.1. So, we must have that $e\left(\left\{\hat{\tau}_{1}, \hat{\tau}_{2}\right\}, X\right)_{U}=\emptyset$. Then $e\left(X, U_{V} \backslash X\right)_{U}=\emptyset$ because $e(X, Y)_{U}=\emptyset$, a contradiction by Lemma 2.1. Hence, we must have $\left\{\hat{\tau}_{1}, \hat{\tau}_{2}\right\} \nsubseteq Y^{\prime}$. Without loss of generality, assume $\hat{\tau}_{1} \in X^{\prime}$ and $\hat{\tau}_{2} \in Y^{\prime}$ in $U$. If $\hat{\tau}_{2} \sigma_{t} \in U$ for some $\sigma_{t} \in X$, then $\hat{\tau_{2}} \sigma_{t} \rightarrow \sigma_{1} \sigma_{t}$ or 
$\hat{\tau_{2}} \sigma_{1}$ and furthermore if $\hat{\tau_{2}} \sigma_{t} \rightarrow \sigma_{1} \sigma_{t}$, then $\sigma_{1} \sigma_{t} \rightarrow \sigma_{1} \hat{\tau_{1}}$ or $\hat{\tau_{1}} \sigma_{t}$ to obtain $U^{\prime} \in \mathcal{Q}(H)$ because $\sigma_{1} \wedge \sigma_{t} \leq \hat{\tau}_{1} \wedge \hat{\tau}_{2}$. Hence $e\left(\hat{\tau}_{2}, X\right)_{U^{\prime}}=\emptyset$ but there is no circuit containing edges $\hat{\tau_{1}} \sigma_{t}$ and $\hat{\tau_{2}} \sigma_{m}$ for $\sigma_{t} \in X, \sigma_{m} \in Y$ in $U^{\prime}$ because $e\left(\left\{\hat{\tau_{1}}, \hat{\tau_{2}}\right\} \cup Y, X\right)_{U^{\prime}}=e\left(\hat{\tau_{1}}, X\right)_{U^{\prime}}$, a contradiction. Hence, we must conclude that $\hat{\tau_{2}} \in X^{\prime}$. Thus, we proved that $\left\{\hat{\tau}_{1}, \hat{\tau_{2}}\right\} \cup X \subseteq X^{\prime}$. By symmetry we can also show that if $A_{1}=\emptyset$, then $\left\{\hat{\tau}_{1}, \hat{\tau_{2}}\right\} \cup X \subseteq Y^{\prime}$ and similarly if $\left\{\sigma_{1}, \sigma_{2}\right\} \subseteq X$, then $\left\{\hat{\tau}_{1}, \hat{\tau_{2}}\right\} \cup Y \subseteq Y^{\prime}$ or $X^{\prime}$.

(b) Suppose $\sigma_{1}=\hat{\tau}_{1}, \sigma_{2} \neq \hat{\tau}_{2}$ and $\sigma_{2} \in Y$ in $U$. Let $A_{1}=X \cap X^{\prime}$ and $A_{2}=X \cap Y^{\prime}$ and suppose $A_{1} \neq \emptyset$ and $A_{2} \neq \emptyset$. If $\hat{\tau}_{2} \in X^{\prime}$ (respectively, $Y^{\prime}$ ) and $\hat{\tau}_{2} \sigma_{t} \in U$, then $\hat{\tau}_{2} \sigma_{t} \rightarrow \hat{\tau}_{1} \sigma_{t}$ or $\hat{\tau}_{1} \hat{\tau}_{2}$ for all $\sigma_{t} \in A_{2}$ (respectively, $A_{1}$ ) and furthermore if $\hat{\tau}_{2} \sigma_{t} \rightarrow \hat{\tau}_{1} \hat{\tau}_{2}$, then $\hat{\tau}_{1} \hat{\tau}_{2} \rightarrow \hat{\tau}_{1} \sigma_{2}$ or $\sigma_{2} \hat{\tau}_{2}$ because $\hat{\tau}_{1} \wedge \hat{\tau}_{2}=\hat{\tau}_{2} \wedge \sigma_{t} \leq$ $\hat{\tau}_{1} \wedge \sigma_{2}$. So, there exists $U^{\prime} \in \mathcal{Q}(H)$ such that (i) $e\left(\hat{\tau}_{2}, A_{1}\right)_{U^{\prime}}=\emptyset$ if $\hat{\tau}_{2} \in Y^{\prime}$ and $e\left(\hat{\tau}_{2}, A_{2}\right)_{U^{\prime}}=\emptyset$ if $\hat{\tau}_{2} \in X^{\prime}$ (ii) $\hat{\tau}_{1} \hat{\tau}_{2} \notin U^{\prime}$ (iii) $e\left(A_{1}, A_{2}\right)_{U^{\prime}}=\emptyset$ and $e(X, Y)_{U^{\prime}}=\emptyset$. Then for some $\sigma_{t} \in Y, \sigma_{k} \in A_{1}, \sigma_{m} \in A_{2}$ there is no circuit in $U^{\prime}$ containing edges $\hat{\tau}_{2} \sigma_{t}$ and $\hat{\tau}_{1} \sigma_{k}$ if $\hat{\tau}_{2} \in Y^{\prime}$ because $e\left(A_{1},\left(U^{\prime}\right)_{V} \backslash A_{1}\right)_{U^{\prime}}=e\left(\hat{\tau}_{1}, A_{1}\right)_{U^{\prime}}$, a contradiction. Similarly, we can show that if $\hat{\tau}_{2} \in X^{\prime}$, then there is no circuit containing $\hat{\tau}_{2} \tau_{t}$ and $\sigma_{1} \sigma_{m}$. So, we must have $A_{1}=\emptyset$ or $A_{2}=\emptyset$. Without loss of generality, let $A_{2}=\emptyset$ and assume $X \subseteq X^{\prime}$ in $U$ and with a similar argument as above we can show $\hat{\tau_{2}} \in X^{\prime}$, hence $\left\{\hat{\tau_{2}}\right\} \cup X \subseteq X^{\prime}$. With a symmetric argument we can show that if $A_{1}=\emptyset$, then $\left\{\hat{\tau}_{2}\right\} \cup X \subseteq Y^{\prime}$ and similarly if $\sigma_{2} \in X$, then $\left\{\hat{\tau_{2}}\right\} \cup Y \subseteq Y^{\prime}$ or $X^{\prime}$.

Lemma 2.3. Let $H=\mathcal{G}\left(\sigma_{1}, \ldots, \sigma_{n}\right)=\mathcal{G}\left(\hat{\tau}_{1}, \hat{\tau}_{2}, \tau_{3}, \ldots, \tau_{n}\right)=\left(G ; X, Y ; \tau_{1}, \tau_{2}\right)$ be strongly indecomposable. If $X^{\prime}, Y^{\prime}$ is a TVE-partition for $\sigma_{i}, \sigma_{j}$ in $H$, then there exists $U \in \mathcal{Q}(H)$ such that $e(X, Y)_{U}=\emptyset$ and $e\left(X^{\prime}, Y^{\prime}\right)_{U}=\emptyset$.

Proof. For notational convenience, after rearranging the indices of $H$, we let $\sigma_{1}=\sigma_{i}$ and $\sigma_{2}=\sigma_{j}$. By Lemma 1.4 there exists $V \in \mathcal{Q}(H)$ such that edge $\sigma_{k} \sigma_{m} \notin V$ if $\sigma_{k} \wedge \sigma_{m} \leq \hat{\tau}_{1} \wedge \hat{\tau_{2}}$ for $3 \leq k, m \leq n$ and edge $\tau_{k} \tau_{m} \notin T$ for all $\tau_{k} \wedge \tau_{m} \leq \tau_{t}$ where $\tau_{k}, \tau_{m} \in X$ and $\tau_{t} \in Y$. So, in particular $e(X, Y)_{V}=\emptyset$.

Throughout the proof, we let $\sigma_{k} \in X^{\prime}$ and $\sigma_{m} \in Y^{\prime}$. We will consider the following cases: (i) If $\sigma_{t}=\hat{\tau}_{t}$ for $t=1,2$, then we let $U=V$. (ii) If $\sigma_{1}=\hat{\tau_{1}}, \sigma_{2} \neq \hat{\tau_{2}}$ and if edge $\sigma_{k} \sigma_{m} \in V$, then we can $\sigma_{k} \sigma_{m} \rightarrow \sigma_{2} \sigma_{k}$ or $\sigma_{2} \sigma_{m}$ by Lemma 2.2(b). (iii) If $\sigma_{1} \in X, \sigma_{2} \in Y$ and edge $\sigma_{k} \sigma_{m} \in V$, then $\sigma_{k} \sigma_{m} \rightarrow \sigma_{1} \sigma_{k}$ or $\sigma_{1} \sigma_{m}$ if $\sigma_{k}, \sigma_{m} \in X$ and $\sigma_{k} \sigma_{m} \rightarrow \sigma_{2} \sigma_{k}$ or $\sigma_{2} \sigma_{m}$ if $\sigma_{k}, \sigma_{m} \in Y$. If $\hat{\tau}_{1} \in X^{\prime}, \hat{\tau_{2}} \in Y^{\prime}$ and edge $\hat{\tau}_{1} \hat{\tau_{2}} \in V$, then $\hat{\tau}_{1} \hat{\tau_{2}} \rightarrow \hat{\tau}_{1} \sigma_{1}$ or $\hat{\tau}_{2} \sigma_{1}$. If $\hat{\tau}_{r} \in X^{\prime}$ for $r \in\{1,2\}$ and $\sigma_{m} \in Y^{\prime} \cap X$ (respectively, $Y^{\prime} \cap Y$ ) and edge $\hat{\tau}_{r} \sigma_{m} \in V$, then $\hat{\tau}_{r} \sigma_{m} \rightarrow \sigma_{1} \sigma_{m}$ or $\sigma_{1} \hat{\tau}_{r}$ (respectively, $\hat{\tau}_{r} \sigma_{m} \rightarrow \sigma_{2} \sigma_{m}$ or $\sigma_{2} \hat{\tau}_{r}$ ). (iv) By Lemma 2.2(a), if $\left\{\sigma_{1}, \sigma_{2}\right\} \subseteq X$ or $Y$ and edge $\sigma_{k} \sigma_{m} \in V$, then $\sigma_{k} \sigma_{m} \rightarrow \sigma_{r} \sigma_{k}$ or $\sigma_{r} \sigma_{m}$ for $r \in\{1,2\}$. Therefore, there exists $U \in \mathcal{Q}(H)$ such that $e\left(X^{\prime}, Y^{\prime}\right)_{U}=\emptyset$ and $e(X, Y)_{U}=\emptyset$.

Let $G=\mathcal{G}\left(\tau_{1}, \ldots, \tau_{n}\right)$ and $H=\mathcal{G}\left(\sigma_{1}, \ldots, \sigma_{n}\right)$. If $\tau_{i}=\sigma_{j}$ for some $j$, then we say $\tau_{i}$ is a common vertex in $G$ and $H$. We say that there is a sequence of two-vertex exchanges transforming $G$ to $H$ if there is a sequence of groups 
$G=G_{1}, G_{2}, \ldots, G_{n}=H$ such that $G_{i+1}$ is obtained from $G_{i}$ by a two-vertex exchange and common vertices of $G$ and $H$ are not replaced by each two-vertex exchange. Some results on the sequence of two-vertex exchanges to transform a group to a quasi-isomorphic group can be found in [8].

Corollary 2.4. Let $G=\mathcal{G}\left(\tau_{1}, \ldots, \tau_{n}\right)$ be strongly indecomposable and suppose $H$ is obtained by a sequence of two two-vertex exchanges from $G$. Then there is a CLP-map $\psi: T \rightarrow U$ for some $T \in \mathcal{Q}(G)$ and $U \in \mathcal{Q}(H)$.

Proof. Suppose $H$ is obtained by a sequence of two two-vertex exchanges from $G$ and let $G_{1}=\left(G ; X_{1}, Y_{1} ; \tau_{1}, \tau_{2}\right)$ and $H=\left(G_{1} ; X_{2}, Y_{2} ; \sigma_{i}, \sigma_{j}\right)$. Then there exists $V \in \mathcal{Q}\left(G_{1}\right)$ such that $e\left(X_{1}, Y_{1}\right)_{V}=\emptyset$ and $e\left(X_{2}, Y_{2}\right)_{V}=\emptyset$ by Lemma 2.3. Let $\phi: \mathcal{C}_{G_{1}} \rightarrow \mathcal{C}_{G}$ and $\psi: \mathcal{C}_{G_{1}} \rightarrow \mathcal{C}_{H}$ be TVE-maps then $T=\phi(V) \in \mathcal{Q}(G)$ and $U=\psi(V) \in \mathcal{Q}(H)$, and $\left.\phi^{-1}\right|_{T}: T \rightarrow V$ and $\left.\psi\right|_{V}: V \rightarrow U$ are CLP-maps by Proposition 1.5(a). Hence $\left(\left.\psi\right|_{V}\right) \circ\left(\left.\phi^{-1}\right|_{T}\right): T \rightarrow U$ is the desired CLP-map.

Corollary 2.4 provides an induction step to prove Lemma 2.5.

Lemma 2.5. Let $G=\mathcal{G}\left(\tau_{1}, \ldots, \tau_{n}\right)$ and $H=\mathcal{G}\left(\sigma_{1}, \ldots, \sigma_{n}\right)$ be strongly indecomposable with both $\tau_{1}, \ldots, \tau_{n}$ and $\sigma_{1}, \ldots, \sigma_{n}$ trimmed. Then the following are equivalent:

(a) $G \dot{\simeq} H$

(b) There is a sequence of two-vertex exchanges transforming $G$ to $H$;

(c) There is a CLP-map $\psi: T \rightarrow U$ for some $T \in \mathcal{Q}(G)$ and $U \in \mathcal{Q}(H)$.

Proof. (a) $\Rightarrow$ (b) Theorem 2.3 in $[6]$.

(b) $\Rightarrow$ (c) We will prove the existence of a CLP-map $\psi: T \rightarrow U$ for some $T \in \mathcal{Q}(G)$ and $U \in \mathcal{Q}(H)$ by the induction on $m=$ the number of twovertex exchanges to transform $G$ to $H$. If $m \leq 2$, then there is a CLP-map $\psi: T \rightarrow U$ for some $T \in \mathcal{Q}(G)$ and $U \in \mathcal{Q}(H)$ by Proposition $1.5($ a) if $m=1$ and by Corollary 2.4 if $m=2$. So, assume it is true for $m \leq n-1$ and suppose there is a sequence of $n$ two-vertex exchanges transforming $G$ to $H$. Let $G=G_{1}, \ldots, G_{n}, G_{n+1}=H$ such that $G_{i+1}$ is obtained from $G_{i}$ by a twovertex exchange. Without loss of generality we define $G_{1}=\mathcal{G}\left(\tau_{1}^{1}, \tau_{2}^{1}, \ldots, \tau_{n}^{1}\right)$ and $G_{i+1}=\left(G_{i} ; X_{i}, Y_{i} ; \tau_{1}^{i}, \tau_{2}^{i}\right)$, where $X_{i}, Y_{i}$ is the TVE-partition for $\tau_{1}^{i}, \tau_{2}^{i}$ in $G_{i}=\mathcal{G}\left(\tau_{1}^{i}, \tau_{2}^{i}, \ldots, \tau_{n}^{i}\right)$ for $i=1, \ldots, n$. Let $\phi_{i}: \mathcal{C}_{G_{i}} \rightarrow \mathcal{C}_{G_{i+1}}$ be the TVE-map for each $i$ and define $E_{1}=\left\{\tau_{i}^{1} \tau_{j}^{1}: \tau_{i}^{1} \tau_{j}^{1}\right.$ is an edge in $\mathcal{C}_{G_{1}}$ and $\tau_{i}^{1} \in X_{1}$ and $\left.\tau_{j}^{1} \in Y_{1}\right\}$ and $E_{i+1}=\phi_{i}\left(E_{i}\right)$ inductively for $i=1, \ldots, n$ (Recall that a quasirepresenting graph for $G$ is any subgraph of $\mathcal{C}_{G}$ that is obtained by iteration of the algorithm: if a graph contains a circuit $S$ with all the edges labelled by types $\geq \tau$ and at least one edge labelled by $\tau$, then remove an edge of $S$ labelled by $\tau$ ).

We first show that $\mathcal{C}_{G_{i}}^{\prime}=\mathcal{C}_{G_{i}} \backslash E_{i}$ contains a quasi-representing graph for $G_{i}$ for each $i$. Let $e_{1}=\tau_{i}^{1} \tau_{j}^{1}$ be an arbitrary edge in $E_{1}$. If $f_{1}=\tau_{i}^{1} \tau_{1}^{1}$ is an edge incident to $\tau_{1}^{1}$ in $\mathcal{C}_{G_{1}}$, then label $e_{1} \leq$ label $f_{1}$ and define $e_{i+1}=\phi_{i}\left(e_{i}\right)$ and $f_{i+1}=\phi_{i}\left(f_{i}\right)$ inductively for $i=1, \ldots, n$. Since $\phi_{i}$ is the TVE-map preserving 
labels of edges, it follows that label $e_{i}=$ label $e_{1}$ and label $f_{i}=$ label $f_{1}$ and label $e_{i} \leq$ label $f_{i}$ for $i=2, \ldots, n+1$. Suppose edge $f_{i}$ is incident to some vertex $\tau_{k}^{i}$ in $\mathcal{C}_{G_{i}}$ then $\tau_{k}^{i} \geq$ label $f_{i}$ and $\tau_{k}^{i} \geq$ label $e_{i}$. So, if edge $e_{i}=\tau_{s}^{i} \tau_{t}^{i}$ in $\mathcal{C}_{G_{i}}$, then $\tau_{k}^{i}, \tau_{s}^{i}, \tau_{t}^{i}$ is a circuit with edges $\tau_{k}^{i} \tau_{s}^{i}, \tau_{s}^{i} \tau_{t}^{i}, \tau_{t}^{i} \tau_{k}^{i}$ labelled with types $\geq \tau_{s}^{i} \wedge \tau_{t}^{i}$. Thus, we can remove $e_{i}=\tau_{s}^{i} \tau_{t}^{i}$ from $\mathcal{C}_{G_{i}}$ and $\mathcal{C}_{G_{i}} \backslash\left\{e_{i}\right\}$ contains a quasi-representing graph for $G_{i}$. Similarly, we can remove all other edges in $E_{i}$ from $\mathcal{C}_{G_{i}}$, hence we showed that $\mathcal{C}_{G_{i}}^{\prime}=\mathcal{C}_{G_{i}} \backslash E_{i}$ contains a quasi-representing graph for $G_{i}$ for $i=1, \ldots, n+1$.

By the induction hypothesis on $m$, there exists $T \in \mathcal{Q}(H)$ such that $T \subseteq$ $\mathcal{C}_{G_{n+1}}^{\prime}$ and $V \in \mathcal{Q}\left(G_{2}\right)$ such that $\psi^{\prime}: T \rightarrow V$ is a CLP-map. Since $\psi^{\prime}$ is a bijection map between edges of $T$ and edges of $V$, it follows that $V \subseteq \mathcal{C}_{G_{2}}^{\prime}$ and $e\left(X_{1}, Y_{1}\right)_{V}=\emptyset$ because $\tau_{r}^{i+1}=\tau_{r}^{i}$ for $r=3, \ldots, n$ by Proposition 1.5(b). Hence, by Proposition 1.5(a), $U=\phi_{1}^{-1}(V) \in \mathcal{Q}\left(G_{1}\right)$ and $\psi=\left.\phi_{1}^{-1}\right|_{V}: V \rightarrow U$ is a CLP-map, where $\phi_{1}: \mathcal{C}_{G_{1}} \rightarrow \mathcal{C}_{G_{2}}$ is a TVE-map. The map $\psi \circ \psi^{\prime}: T \rightarrow U$ is the desired CLP-map.

(c) $\Rightarrow$ (a) Lemma 1.2.

A group is completely decomposable if it is a direct sum of rank one summands. Two completely decomposable finite rank torsion-free abelian groups are quasi-isomorphic if and only if they have an equal number of quasi-summands of same type for all types (or equivalently, two completely decomposable groups are quasi-isomorphic if and only if their quasi-representing graphs have equal numbers of edges of same types for all types). Since $G$ is completely decomposable if and only if $T \in \mathcal{Q}(G)$ is a tree by Corollary 1.9 in [1], it is clear that any label-preserving bijection between the edges of two trees is a CLP-map. Hence, we proved that:

Lemma 2.6. Let $G=\mathcal{G}\left(\tau_{1}, \ldots, \tau_{n}\right)$ and $H=\mathcal{G}\left(\sigma_{1}, \ldots, \sigma_{n}\right)$ be completely decomposable groups. If $G \dot{\sim} H$, then there is a CLP-map $\psi: T \rightarrow U$ for some $T \in \mathcal{Q}(G)$ and $U \in \mathcal{Q}(H)$.

If $G$ is decomposable group, then $G \dot{\sim} G_{1} \oplus \cdots \oplus G_{k}$ where each $G_{i}$ is either completely decomposable or strongly indecomposable of rank $>1$ and each $G_{i}$ has a quasi-representing graph $T_{i}$ and $T_{i} \cap T_{j}$ does not contain an edge if $i \neq j$ and $T=\bigcup_{i} T_{i} \in \mathcal{Q}(G)$.

We next show that the strongly indecomposability condition can be removed in Lemma 2.5 .

Theorem 2.7. Let $G=\mathcal{G}\left(\tau_{1}, \ldots, \tau_{n}\right)$ and $H=\mathcal{G}\left(\sigma_{1}, \ldots, \sigma_{n}\right)$. Then $G \dot{\simeq} H$ if and only if there is a CLP-map $\psi: T \rightarrow U$ for some $T \in \mathcal{Q}(G)$ and $U \in \mathcal{Q}(H)$.

Proof. Suppose $G \dot{\simeq} H$. By Lemma 2.5, we assume $G$ is decomposable. Let $G \dot{\sim} G_{1} \oplus \cdots \oplus G_{k}$ and $H \dot{\sim} H_{1} \oplus \cdots \oplus H_{m}$, where each $G_{i}$ and $H_{j}$ are either completely decomposable or strongly indecomposable of rank $>1$. Since $G \dot{\simeq} H$ it follows that $k=m$ and, without loss of generality, assume $G_{i} \dot{\sim} H_{i}$ and there exists $T_{i} \in \mathcal{Q}\left(G_{i}\right)$ such that $\psi_{i}: T_{i} \rightarrow U_{i}$ is a CLP-map, where 
$U_{i}=\psi_{i}\left(T_{i}\right) \in \mathcal{Q}\left(H_{i}\right)$ for each $1 \leq i \leq k$. Let $T=\bigcup_{i} T_{i} \in \mathcal{Q}(G)$ and $U=\bigcup_{i} U_{i} \in \mathcal{Q}(H)$ and define a map $\psi: T \rightarrow U$ such that $\left.\psi\right|_{T_{i}}=\psi_{i}$ for each $1 \leq i \leq k$, then $\psi$ is the desired CLP-map.

The converse is clear by Lemma 1.2.

If there is a permutation $\rho$ of $\{1, \ldots, n\}$ such that $\tau_{1}=\sigma_{\rho(1)}, \ldots, \tau_{n}=\sigma_{\rho(n)}$, then we say $\mathcal{G}\left(\tau_{1}, \ldots, \tau_{n}\right)$ and $\mathcal{G}\left(\sigma_{1}, \ldots, \sigma_{n}\right)$ are equivalent. It is easy to see that any two equivalent groups are quasi-isomorphic. We say $G$ is an elementary group if $T(\tau)$ is either emptyset, a singleton edge or $T$ for all types $\tau$, where $T \in \mathcal{Q}(G)$.

We say a subgraph $B \subseteq T$ is a block if $B$ is the intersection of all circuits in $T$ containing $B$. It is easy to see that if $\psi: T \rightarrow U$ is a CLP-map, then $B$ is a block in $T$ if and only if $\psi(B)$ is a block in $U$.

Theorem 2.8. There are at most $\frac{(n-1) !}{2}$ non-equivalent groups quasi-isomorphic to strongly indecomposable group $\mathcal{G}\left(\tau_{1}, \ldots, \tau_{n}\right)$, where $n \geq 3$.

Proof. Let $G=\mathcal{G}\left(\tau_{1}, \ldots, \tau_{n}\right)$ be a strongly indecomposable group and $T \in$ $\mathcal{Q}(G)$. Note that since a TVE-partition gives a non-equivalent quasi-isomorphic group by Lemma 1.1, we investigate which groups provide the maximum number of TVE-partitions. By the remark following Theorem 7 in [7] the necessary and sufficient conditions for a TVE-partition $X, Y$ for $\tau_{1}, \tau_{2}$ using a quasirepresenting graph are $e(X, Y)_{T}=\emptyset$ and $\tau^{X} \wedge \tau^{Y} \leq \tau_{1} \wedge \tau_{2}$ and observe that if $\psi: T \rightarrow U$ is a CLP-map, then $U$ is obtained by permuting labels of edges of blocks in $T$ because $\psi$ sends a circuit to a circuit. Hence, we get the maximum number of permutations of labels of edges in $T$ if there is least number of blocks in $T$. That is, we obtain the maximum number of non-equivalent quasi-isomorphic groups if $T$ is a circuit. So, we assume $T$ is a circuit. If $G$ is an elementary group, then $X, Y$ is a TVE-partition if and only if $e(X, Y)_{T}=\emptyset$ by Lemma 8 in [7]. Hence, we assume $G$ is elementary and $T$ is a circuit, then there are exactly $\frac{(n-1) !}{2}$ non-equivalent elementary groups quasi-isomorphic to $G$ by Corollary 10 in [7]. Thus, for an arbitrary strongly indecomposable group $G$ there are at most $\frac{(n-1) !}{2}$ non-equivalent groups quasi-isomorphic to $G$.

Define $\mathcal{G}\left[A_{1}, \ldots, A_{n}\right]=$ the cokernel of the diagonal embedding $\bigcap_{i=1}^{n} A_{i} \rightarrow$ $\bigoplus_{i=1}^{n} A_{i}$, then $\mathcal{G}\left[A_{1}, \ldots, A_{n}\right]$ is a $\mathcal{B}^{(1)}$-group and the class of groups $\mathcal{G}\left[A_{1}, \ldots\right.$, $\left.A_{n}\right]$ is the dual class of groups $\mathcal{G}\left(A_{1}, \ldots, A_{n}\right)$ in the sense of quasi-isomorphism Butler duality of [4]. Let $G=\mathcal{G}\left[\tau_{1}, \ldots, \tau_{n}\right]$ and define $\overline{\mathcal{C}}_{G}$ be the complete graph with vertices $\tau_{1}, \ldots, \tau_{n}$ and edges $\tau_{i} \tau_{j}$ labelled by types $\tau_{i} \vee \tau_{j}$ for $1 \leq$ $i \neq j \leq n$. A co-representing graph for $G$ is any subgraph of $\overline{\mathcal{C}}_{G}$ that is obtained by iteration of the algorithm: if a graph contains a circuit $S$ with all the edges labelled by types $\leq \tau$ and at least one edge labelled by $\tau$, then remove an edge of $S$ labelled by $\tau$. Let $\mathcal{C Q}(G)$ be the set of all co-quasi-representing graphs for $G$. 
The following corollary can be obtained using the quasi-isomorphism Butler duality of [4].

Corollary 2.9. Let $G=\mathcal{G}\left[\tau_{1}, \ldots, \tau_{n}\right]$ and $H=\mathcal{G}\left[\sigma_{1}, \ldots, \sigma_{n}\right]$.

(a) $G \dot{\sim} H$ if and only if there is a CLP-map $\psi: T \rightarrow U$ for some $T \in$ $\mathcal{C} \mathcal{Q}(G)$ and $U \in \mathcal{C} \mathcal{Q}(H)$.

(b) There are at most $\frac{(n-1) !}{2}$ non-equivalent groups quasi-isomorphic to strongly indecomposable group $\mathcal{G}\left[\tau_{1}, \ldots, \tau_{n}\right]$, where $n \geq 3$.

\section{References}

[1] D. Arnold and C. Vinsonhaler, Representing graphs for a class of torsion-free abelian groups, Abelian Group Theory (Oberwolfach, 1985), 309-332, Gordon and Breach, New York, 1987.

[2] _ Quasi-isomorphism invariants for a class of torsion-free abelian groups, Houston J. Math. 15 (1989), no. 3, 327-340.

[3] - Invariants for a class of torsion-free abelian groups, Proc. Amer. Math. Soc. 105 (1989), no. 2, 293-300

[4] — Duality and Invariants for Butler groups, Pacific J. Math. 148 (1991), no. 1, $1-9$.

[5] F. Richman, An extension of the theory of completely decomposable torsion-free abelian groups, Trans. Amer. Math. Soc. 279 (1983), no. 1, 175-185.

[6] P. Yom, A characterization of a class of Butler groups, Comm. Algebra 25 (1997), no. $12,3721-3734$.

[7] _ A characterization of a class of Butler groups II, Abelian group theory and related topics (Oberwolfach, 1993), 419-432, Contemp. Math., 171, Amer. Math. Soc., Providence, RI, 1994.

[8] _ A relationship between vertices and quasi-isomorphism for a class of bracket groups, J. Korean Math. Soc. 44 (2007), no. 6, 1197-1211.

Department of Mathematics And Computer Science

Bronx Community College of CUNY

Bronx, 10453, New York, USA

E-mail address: peter.yom@bcc.cuny.edu 